\title{
Biodegradable polyesters reinforced with triclosan loaded polylactide micro/nanofibers: Properties, release and biocompatibility
}

\author{
L. J. del Valle, * A. Díaz, M. Royo, A. Rodríguez-Galán, J. Puiggalí \\ Departament d'Enginyeria Química, Universitat Politècnica de Catalunya, Av. Diagonal 647, E-08028 Barcelona, Spain
}

Received 14 July 2011; accepted in revised form 19 October 2011

\begin{abstract}
Mechanical properties and drug release behavior were studied for three biodegradable polyester matrices (polycaprolactone, poly(nonamethylene azelate) and the copolymer derived from 1,9-nonanediol and an equimolar mixture of azelaic and pimelic acids) reinforced with polylactide (PLA) fibers. Electrospinning was used to produce suitable mats constituted by fibers of different diameters (i.e. from micro- to nanoscale) and a homogeneous dispersion of a representative hydrophobic drug (i.e. triclosan). Fabrics were prepared by a molding process, which allowed cold crystallization of PLA micro/nanofibers and hot crystallization of the polyester matrices. The orientation of PLA molecules during electrospinning favored the crystallization process, which was slightly enhanced when the diameter decreased. Incorporation of PLA micro/nanofibers led to a significant increase in the elastic modulus and tensile strength, and in general to a decrease in the strain at break. The brittle fracture was clearer when high molecular weight samples with high plastic deformation were employed.

Large differences in the release behavior were detected depending on the loading process, fiber diameter size and hydrophobicity of the polyester matrix. The release of samples with the drug only loaded into the reinforcing fibers was initially fast and then became slow and sustained, resulting in longer lasting antimicrobial activity. Biocompatibility of all samples studied was demonstrated by adhesion and proliferation assays using HEp-2 cell cultures.
\end{abstract}

Keywords: biodegradable polymers, reinforcements, mechanical properties, electrospinning, drug release

\section{Introduction}

Polymeric micro/nanofibers are highly interesting materials for applications such as tissue engineering and drug delivery because the large surface area to volume ratio affords interesting new properties [1$3]$. Electrospinning is currently one of the most versatile processes for preparing such kind of fibers since it is applicable to a wide range of materials (e.g. synthetic and natural polymers) at a low cost and with high yield. Basically, electrospinning makes use of electrostatic forces $(10-100 \mathrm{kV})$ to stretch a polymer dilute solution as it solidifies. In addition, this technique provides a huge material elongation rate and significant reduction of the cross-sectional area $[4,5]$ (i.e. close to $1000 \mathrm{~s}^{-1}$ and $10^{5}$, respectively), which may affect the orientation of molecular chains within the fiber and strongly influence the mechanical properties of nanofibers $[6,7]$.

The use of reinforcing fillers and fibers in polymers to improve their mechanical properties is commonly encountered in polymer technology. Properties are strongly dependent on the fiber/matrix interface, and nanofibers should therefore deliver particularly good characteristics due to their high specific surface area. Electrospun fibers are highly promising candidates as reinforcing agents in the develop-

\footnotetext{
${ }^{*}$ Corresponding author, e-mail: luis.javier.del.valle@upc.edu
} (c) BME-PT 
ment of advanced nanocomposites due to their continuity, orientation, inherent flexibility and potential high compatibility with polymer matrices. Thus, in recent years interest has extended to biodegradable materials reinforced with electrospun nanofibers [8-10].

Electrospun fibers can be easily loaded with pharmacologically active agents by adding the appropriate drug to the polymer solution prior to the electrospinning process. In this way, bioresorbable threedimensional tissue scaffolding matrices as drug delivery platforms for therapeutic agents can be prepared. Moreover, results on the ability of different cells to adhere to and proliferate on fibrous scaffolds have encouraged application research in this area [11]. Efficient drug loaded materials must maintain their pharmacological activity throughout a prolonged period of time, the drug release pattern being mainly governed by the interactions between polymer and drug. In this work, we consider the possibility of achieving a sustained release by embedding mats constituted by drug loaded micro/nanofibers into a biodegradable polymeric matrix. In addition to the minimization of the Burst effect and the delay in the release, an improvement of mechanical properties and performance of the matrix is expected. Triclosan (2,4,4'-trichloro-2'-hydroxydiphenyl ether), a well known antimicrobial drug [12], was chosen as a model for studying the release behavior of a hydrophobic drug from polyester matrices having a high methylene content.

Aliphatic polyesters currently constitute the main family of biodegradable polymers despite generally lacking good thermal and mechanical properties. Polylactide (PLA) is a remarkable exception because of its high melting point $\left(173-178^{\circ} \mathrm{C}\right)$ and tensile modulus $(2.7-16 \mathrm{GPa})[13,14]$.

In summary, the main goals of the present work concern to the use of electrospun polylactide micro/ nanofibers as reinforcing materials of biocompatible polyester matrices with low mechanical performance and also to the evaluation of their use as drug delivery platforms to attain a sustained release. Specifically, polycaprolactone (PCL), poly(nonamethylene azelate) (P99) and poly(nonamethylene azelate-co-pimelate) (COP) were selected as matrices due to their different hydrophobicity, crystallinity and synthesis procedures (i.e. ring opening poly- merization or melt polycondensation), which render samples with very different molecular weights.

\section{Experimental section \\ 2.1. Materials}

Triclosan and polycaprolactone $\left(M_{\mathrm{w}}: 65000 \mathrm{~g} / \mathrm{mol}\right)$ were purchased from Sigma-Aldrich (St. Louis, USA). Polylactide, a product of Natureworks ${ }^{\circledR}$ (polymer 2002D), was kindly supplied by Nupik International (Polinyà, Spain). According to the manufacturer, this PLA has a D content of $4.25 \%$, a residual monomer content of $0.3 \%$, a density of $1.24 \mathrm{~g} / \mathrm{cc}$, a glass transition temperature $\left(T_{\mathrm{g}}\right)$ of $58^{\circ} \mathrm{C}$ and a melting point of $153^{\circ} \mathrm{C}$.

Poly(nonamethylene azelate) and poly(nonamethylene azelate-co-pimelate) were synthesized by thermal polycondensation of azelaic acid or a mixture of azelaic and pimelic acids (1:1 molar ratio) with an excess of 1,9-nonanediol (2.2:1 molar ratio), and following a synthetic procedure that was applied for relative polyalkylene dicarboxylate [15-17]. Titanium tetrabutoxyde was used as a catalyst and the reaction was firstly performed in a nitrogen atmosphere at $150^{\circ} \mathrm{C}$ for $6 \mathrm{~h}$ and then in vacuum at $180^{\circ} \mathrm{C}$ for $18 \mathrm{~h}$. Both polymers were purified by precipitation with ethanol of chloroform solutions (10 $\mathrm{wt} \%)$. The polymerization yield was always close to $70 \%$. Weight average molecular weights of 19600 and $38500 \mathrm{~g} / \mathrm{mol}$ were determined by gel permeation chromatography (GPC) (using PMMA standards and 1,1,1,3,3,3-isopropanol as a solvent) for P99 and COP, respectively.

\subsection{Electrospinning and fiber assembly}

Polylactide (PLA) was electrospun from a chloroform-acetone mixture $(2: 1 \mathrm{v} / \mathrm{v})$ using different polymer weight percentage (10-2.5\%). The electrospun fibers were collected on a target placed at different distances from the syringe tip (inside diameter of $0.84 \mathrm{~mm}$ ). The voltage was varied between 10 and $30 \mathrm{kV}$ and applied to the collecting target using a high-voltage supply (Gamma High Voltage Research, ES30-5W) (Ormond Beach, FL, USA). The polymer solutions were delivered via a KDS100 infusion syringe pump from KD Scientific (Holliston, MA, USA) to control the mass-flow rate (from 0.5 to $10 \mathrm{~mL} / \mathrm{h}$ ). All electrospinning experiments were carried out at room temperature. Triclosan 
loaded electrospun fibers were prepared under optimized conditions established for the corresponding polymer solutions. The triclosan weight percentage of these solutions was always $3 \mathrm{wt} \%$.

PCL, P99 and COP matrices were prepared by pressing $(25$ Ton Ring Press, Research \& Industrial Instruments Company, London, UK) approximately $300 \mathrm{mg}$ of the appropriate polymer. The sample was placed into a flat mold covered with teflon sheets to avoid polymer adhesion to the mold. The PCL, P99 and COP samples were pre-heated at $10^{\circ} \mathrm{C}$ above their melting points $\left(60,67\right.$ and $61^{\circ} \mathrm{C}$, respectively) for 3 min and using heating plates and a temperature controller (Graseby Specac, Kent, England). Pressure was progressively increased from 1 to 4 bar. Polymer films with a thickness close to $100 \mu \mathrm{m}$ were recovered after cooling the mold to room temperature.

Matrices loaded with triclosan were prepared in a similar way with a homogeneous mixture previously prepared by solvent-casting. Specifically, $300 \mathrm{mg}$ of the appropriate polymer and $9 \mathrm{mg}(3 \mathrm{wt} \%)$ of triclosan were dissolved in $5 \mathrm{~mL}$ of acetone. The solvent was removed by evaporation at room temperature.

In order to prepare the reinforced matrices, electrospun PLA mats were cut into square pieces with the same lateral dimensions of the previously molded

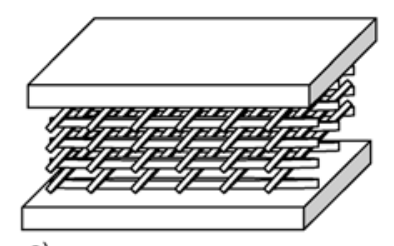

a)
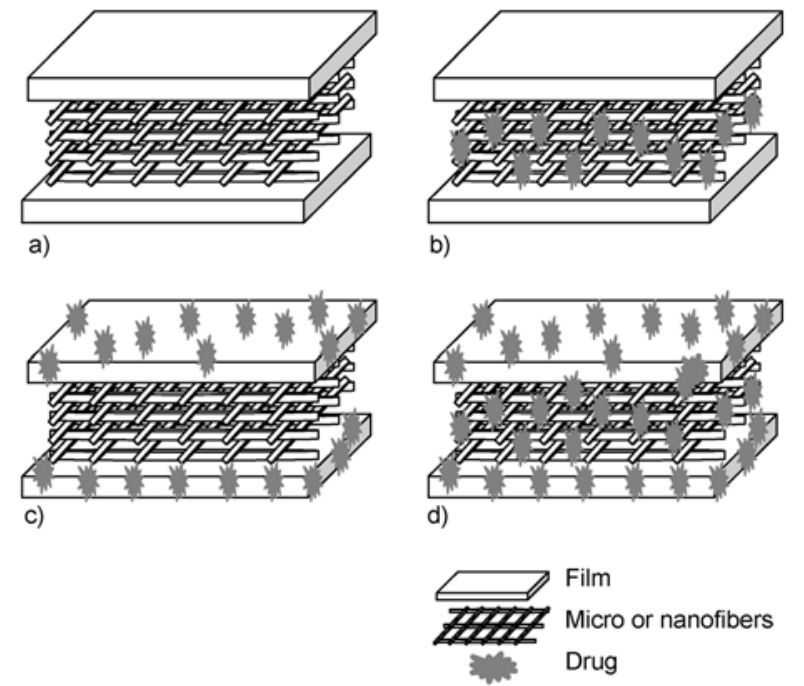

Figure 1. Schematic representation showing samples prepared by melt pressing a mat constituted by PLA micro/nanofibers between two films of PCL, P99 or COP biodegradable polyesters. Samples can be unloaded (a) or loaded with triclosan in the polylactide mat (b), the base polyester films (c) or both the polylactide mat and the base polyester films (d). polymer films. These PLA mats with a weight of ca. $400 \mathrm{mg}$ were placed between the polyester films and pressed following the above indicated procedure (Figure 1). The reinforced material had a PLA weight percentage close to $40 \%$ and a thickness of approximately $330 \mu \mathrm{m}$

\subsection{Measurements}

Molecular weights were estimated by GPC (Shimadzu, model LC-8A) (Tokyo, Japan) equipped with an Empower computer program (Waters Corporation, Massachusetts, USA). A PL HFIP gel column (Polymer Lab) (Agilent Technologies Spain, S.L.; Madrid, Spain) and a refractive index detector (Shimadzu RID-10A) (Tokyo, Japan) were employed.

Optical morphological observations were performed using a Zeiss Axioskop 40 microscope (Carl Zeiss, Göttingen, Germany). Micrographs were taken with a Zeiss AxiosCam MRC5 digital camera. The diameter of the electrospun fibers was measured with a Philips TECNAI 10 transmission electron microscope (TEM) (Philips Electron Optics, Eindhoven, Holland) at an accelerating voltage of $80 \mathrm{kV}$. Fibers were directly electrospun over carbon coated grids. Inspection of fiber surfaces was conducted by scanning electron microscopy (SEM) using a Focus Ion Beam Zeiss Neon 40 instrument (Carl Zeiss, Göttingen, Germany). Carbon coating was accomplished by using a Mitec K950 Sputter Coater (endowed with a film thickness monitor $k 150 \times$ ) (Quorum Technologies Ltd., West Sussex, UK).

Mechanical properties were determined with a Zwick Z2.5/TN1S (Zwick/Roell; Ulm, Germany) testing machine in stress-strain tests carried out at a deformation rate of $10 \mathrm{~mm} / \mathrm{min}$. Measurements were performed on rectangular samples $(30 \times 5 \times$ $0.35 \mathrm{~mm}^{3}$ ) cut from the above melt-pressed pieces. The mechanical parameters were averaged from a minimum of six measurements for each polymer sample.

Contact angles were determined using the water drop method. Images of water drops $(3 \mu 1)$ on the surface of the films were recorded with a contact angle meter (Contact Angle System OCA 15+) (Dataphysics Instruments GmbH, Filderstadt, Germany) after stabilization (30 s). Image analysis and contact angle measurements were carried out with SCA20 
software. Values were averaged for each material over six measurements.

Wide angle X-ray diffraction (WAXD) patterns were acquired at the CRG beamline (BM16) of the European Synchrotron Radiation Facility of Grenoble. The beam was monochromatized to a wavelength of $0.098 \mathrm{~nm}$. Samples were confined between Kapton films. WAXD profiles were acquired in time frames of $12 \mathrm{~s}$ during heating experiments performed at a rate of $3^{\circ} \mathrm{C} / \mathrm{min}$. The WAXD detector was calibrated with diffractions of a standard of an alumina $\left(\mathrm{Al}_{2} \mathrm{O}_{3}\right)$ sample. Diffraction profiles were normalized to the beam intensity and corrected considering the empty sample background. WAXD peaks were deconvoluted with the PeakFit v4 program by Jandel Scientific Software.

\subsection{Release experiments}

Controlled release measurements were made with mats of electrospun samples and polyester/polylactide fabrics cut into square pieces $(10 \times 10 \times$ $0.35 \mathrm{~mm}^{3}$ ). These were weighed and incubated at $37^{\circ} \mathrm{C}$ in an orbital shaker at $60 \mathrm{rpm}$ in vessels with $50 \mathrm{~mL}$ of Sörensen medium (pH 7.4) containing an ethanol volume percentage of $70 \%$. Triclosan concentration in the release medium was evaluated by UV spectroscopy using a UV-3600 (Shimadzu, Tokyo, Japan) UV-Vis-NIR spectrophotometer controlled by the UVProbe 2.31 software. Calibration curves were obtained by plotting the absorbance measured at $283 \mathrm{~nm}$ against triclosan concentration. Samples were drawn from the release medium at predetermined intervals and returned to the release vessel after measuring the absorbance. All drug release tests were carried out using six replicates to control the homogeneity of the release, and the results obtained from the samples were averaged. The total triclosan content in the samples was determined after extraction with chloroform.

\subsection{Cell adhesion and proliferation test}

HEp-2 cells (human laryngeal epidermoid carcinoma) were obtained from ATCC (Virginia, USA). HEp-2 cells were cultured in Dulbecco's Modified Eagle Medium (DMEM) supplemented with $10 \%$ foetal bovine serum, $50 \mathrm{U} / \mathrm{mL}$ penicillin, $50 \mu \mathrm{g} / \mathrm{mL}$ streptomycin and $2 \mathrm{mM} \mathrm{L}$-glutamine at $37^{\circ} \mathrm{C}$ in a humidified atmosphere with $5 \% \mathrm{CO}_{2}$ and $95 \%$ air. The culture medium was changed every two days and, for sub-culture, cell monolayers were rinsed with phosphate buffered saline (PBS) and detached by incubation with trypsin-EDTA $(0.25 \%)$ at $37^{\circ} \mathrm{C}$ for 2-5 min. Cell concentration was determined by count with a Neubauer camera using 4\% vital trypan-blue staining. Detached cells (viability $\geq 95 \%$ ) were cultured under the conditions required for biocompatibility assays.

Electrospun fibers were collected on a glass coverslip (1.4 cm of diameter), and then placed on a 24well culture plate (TCPS) and sterilized by UV irradiation for $15 \mathrm{~min}$. The cells were exposed to direct contact with the material surface for evaluation of cell adhesion and proliferation.

Aliquots of $100 \mu$ containing $5 \cdot 10^{4}$ cells (for adhesion assay) or $2 \cdot 10^{4}$ cells (for proliferation assay) were deposited onto fiber coated disks. The plate was incubated under culture conditions for $60 \mathrm{~min}$ to allow cell attachment to the material surface. Finally, $1 \mathrm{~mL}$ of the culture medium was added to each well. Cell adhesion and proliferation were monitored by culturing cells on the studied surfaces (e.g. TCPS control and polymer matrices).

Cell proliferation was evaluated by MTT (3-(4.5dimethylthiazol-2-yl)-2.5-diphenyl-2H-tetrazolium bromide) after 7 days of culture. Cell adhesion was also evaluated by MTT after $24 \mathrm{~h}$ of culture and the results were normalized to surface unit $\left(\mathrm{cm}^{2}\right)$. Five replicates were averaged and graphically represented for each independent experiment. ANOVA and Tukey tests were performed as statistical analysis at a confidence level of $95 \%(p<0.05)$.

Fiber coated disks were fixed in $2.5 \%$ glutaraldehyde-PBS overnight at $4^{\circ} \mathrm{C}$, dehydrated by washing in an alcohol battery $\left(30,50,70,90,95\right.$ and $\left.100^{\circ}\right)$ at $4^{\circ} \mathrm{C}$ for $30 \mathrm{~min}$ per wash, and finally dyed with Giemsa $1 \%$ for observation in the optical microscope.

\section{Results and discussion}

\subsection{Morphology of electrospun polylactide micro/nanofibers}

Chloroform-acetone mixtures $(2: 1 \mathrm{v} / \mathrm{v})$ with polymer weight percentages of 10 and $2.5 \%$ were successfully assayed and electrospinning parameters were optimized to obtain electrospun fibers with average diameters in the micrometer and nanometer scale (Figure 2), respectively. These diameter sizes were selected to compare reinforcing properties and 

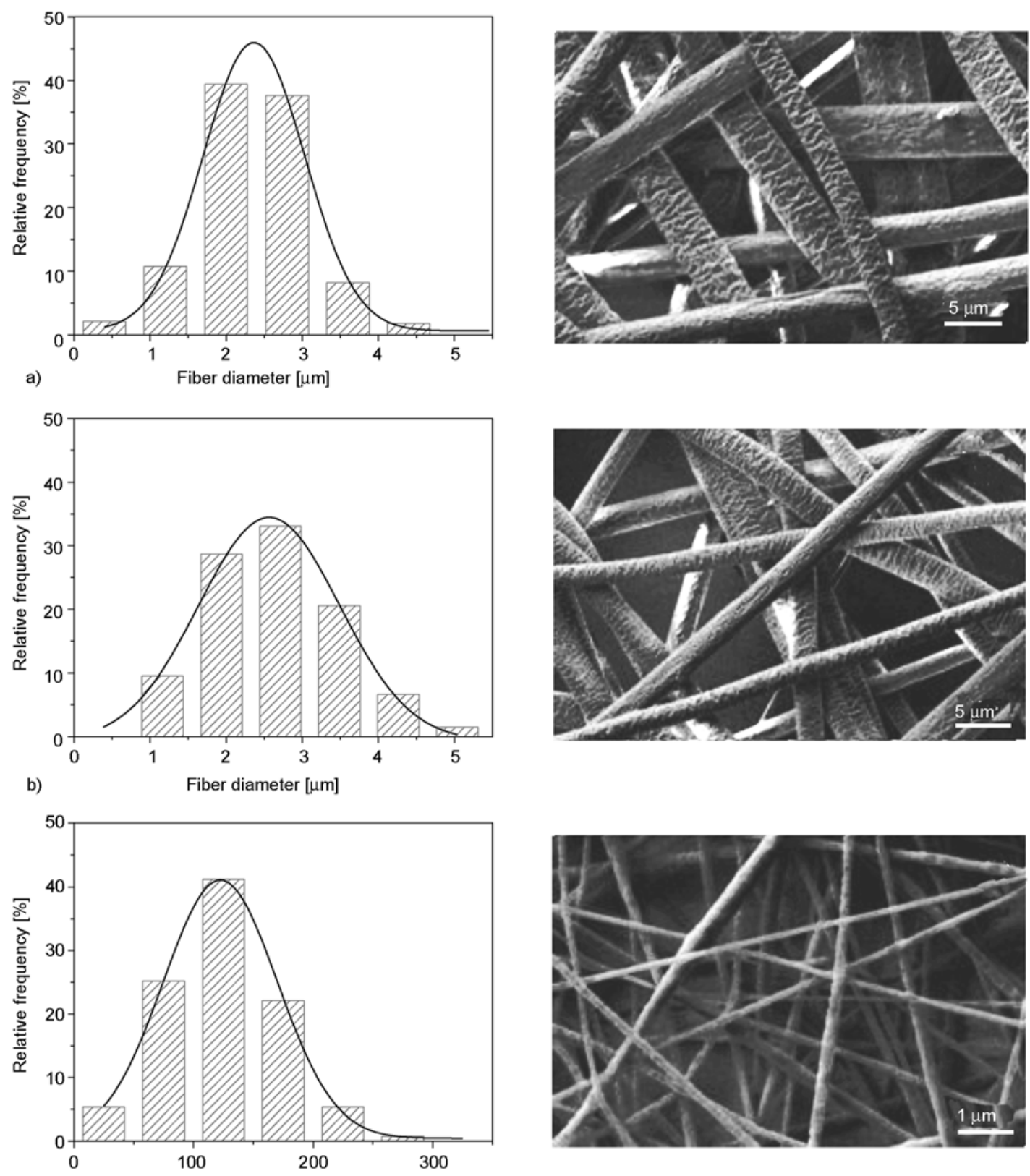

c)

Fiber diameter [nm]
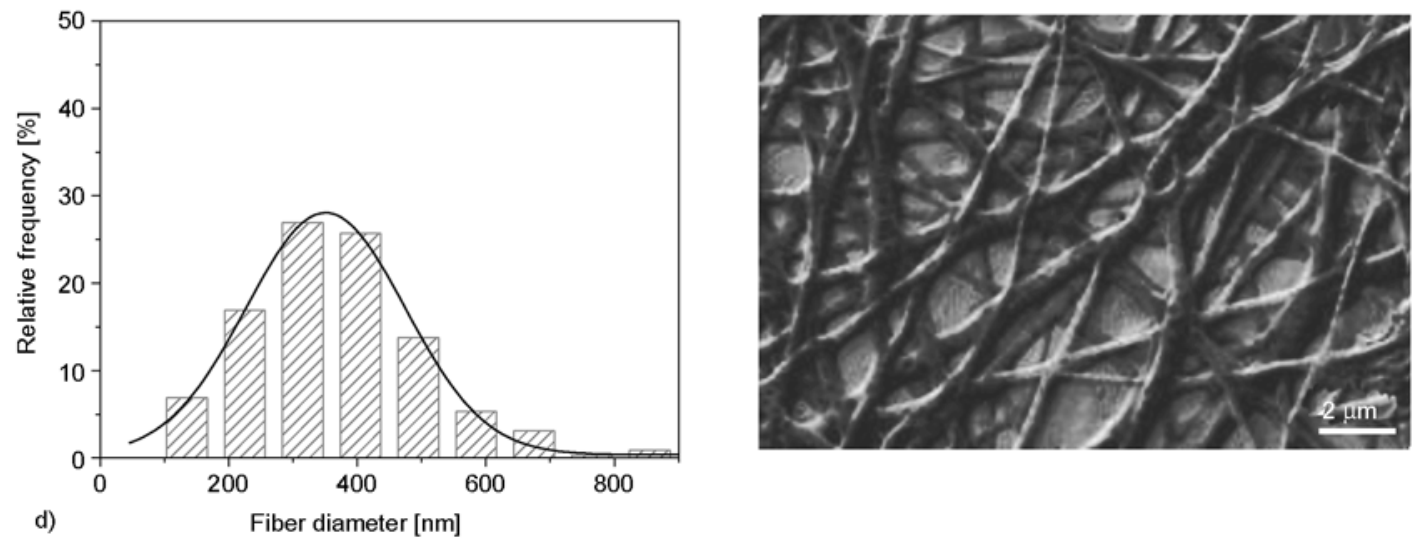

Figure 2. Fiber diameter distribution with Gaussian fitted functions (left) and SEM images (right) of: a) Electrospun PLA microfibers, b) Electrospun PLA microfibers loaded with $3 \mathrm{wt} \%$ of triclosan, c) Electrospun PLA nanofibers, d) Electrospun PLA nanofibers loaded with $3 \mathrm{wt} \%$ of triclosan. 
triclosan release behavior. Accurate fiber diameter distributions were obtained by TEM micrographs using the standard software of the Philips TECNAI 10 electron microscope. Representative histograms of the diameter distributions are given in Figure 2. In all cases, a narrow monomodal distribution was observed and fitted to Gaussian curves which gave average diameters of $2.36 \pm 0.01,2.56 \pm 0.04 \mu \mathrm{m}$ for unloaded and triclosan loaded microfibers and $122 \pm 1$ and $350 \pm 4 \mathrm{~nm}$ for unloaded and triclosan loaded nanofibers. Specifically, the wide diameter range was attained by changing the polymer concentration, whereas tip-collector distance $(12.5 \mathrm{~cm})$, voltage $(15 \mathrm{kV})$ and flow rate $(10 \mathrm{~mL} / \mathrm{h})$ were crucial to obtain homogeneous fibers without electrospraying effect and bead formation. The spinning voltage $(V)$ was carefully selected to ensure a stable conical liquid jet which also minimized bead formation. Note that the surface tension and solution viscosity, which also have a strong influence on fiber morphology, could not be modified since the solvent and polymer concentration remained unchanged for a given micrometer or nanometer diameter size. Once the voltage was optimized, the distance between the target and the syringe tip $(h)$ was varied, and so was the flow rate $(\Phi)$, thus avoiding the coalescence favored by low rates. The diameters of unloaded and triclosan loaded microfibers were highly similar whereas loaded nanofibers were significantly thicker than unloaded ones (i.e. $350 \mathrm{~nm}$ versus

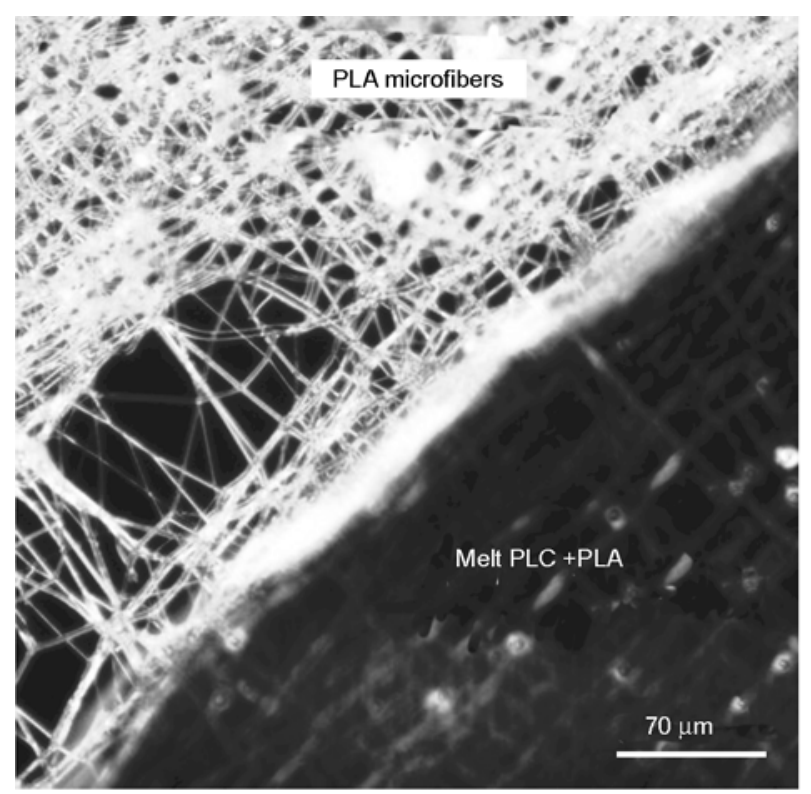

a)
$122 \mathrm{~nm}$ ). SEM micrographs showed that in all cases microfibers had extremely rough surfaces with characteristic transversal striations whereas unloaded nanofibers showed relatively smooth surfaces that became rough with pores when triclosan was incorporated. The texture of microfibers suggests a dense lamellar stacking perpendicular to the fiber axis with amorphous regions between them [18].

\subsection{Crystallinity of polyester matrices reinforced with PLA electrospun micro/nanofibers}

The degree of crystallinity has a great influence on the mechanical properties of polymers and even on their drug release profiles. Thus, it has been found that release can be enhanced by increasing crystallinity, probably due to the increase in drug concentration in the amorphous phase and the partitioning of drug molecules to the sample surface [19]. Specifically, it has been demonstrated that the transformation from amorphous to semicrystalline of a PLA matrix leads to faster drug diffusion through the polymer matrix and an increase of the drug release rate [20]. Hence, the evaluation of the crystallinity of the studied samples is key considering that the preparation method should allow hot crystallization of the polyester matrix (i.e. by slow cooling from the melt state) and indeed cold crystallization of electrospun PLA micro/nanofibers (i.e. by the molding process performed at a temperature

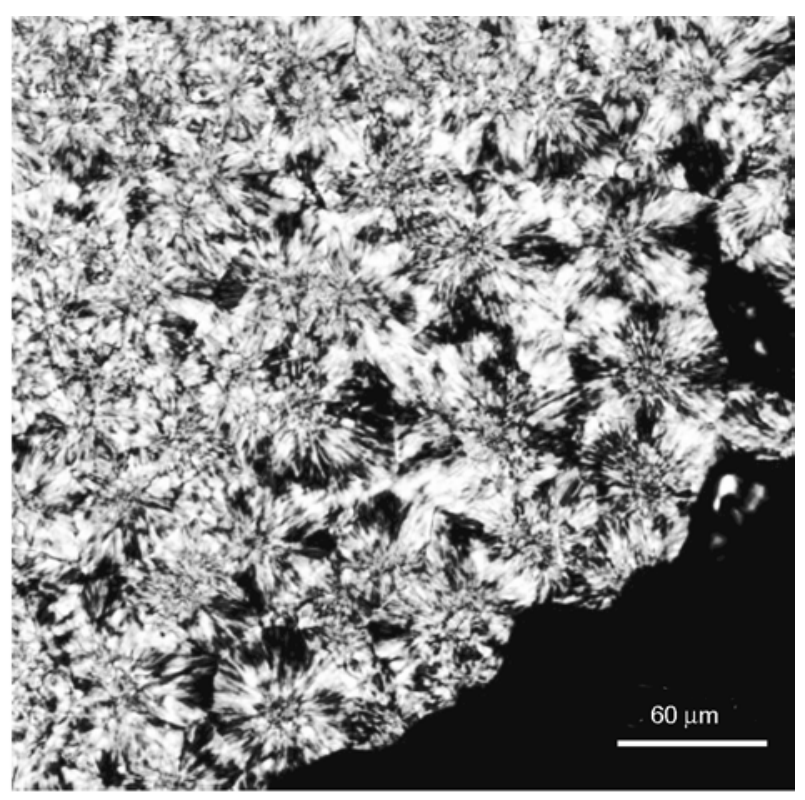

b)

Figure 3. Polarizing optical micrographs showing PLA microfibers embedded into a melted PCL sample (a) and spherulitic morphologies obtained after cooling to room temperature (b) 
higher than the PLA glass transition temperature). Optical micrographs of a fabric of polycaprolactone and electrospun PLA microfibers showed that the highly birefringent microfibers were preserved at the molding temperature and that the melted polyester matrix easily crystallized as negative spherulites when the sample was cooled $\left(3^{\circ} \mathrm{C} / \mathrm{min}\right)$ to room temperature (Figure 3). A similar behavior was observed for preparations involving P99 or COP matrices.

WAXD profiles taken during heating scans of electrospun PLA samples (Figure 4a) showed that these were initially almost amorphous since only two predominant amorphous halos at 0.433 and $0.349 \mathrm{~nm}$ were detected. However, at higher temperatures three Bragg peaks gradually developed at $0.542,0.472$, and $0.403 \mathrm{~nm}$, indicating crystallization of PLA into the well known $\alpha$-form $\left(10_{3}\right.$ helical conformation) since the observed peaks could be indexed as the corresponding (200)+(110), (203) and (015) reflections [21, 22]. Although PLA hardly crystallizes, it is clear that molecular chains attain a high unidirectional orientation during electrospinning, which makes feasible a subsequent reordering process on heating $[23,24]$. The final degree of crystallinity was evaluated from the intensity of the above crystalline and amorphous reflections after deconvolution of the WAXD profile corresponding to the end of the cold crystallization process (Figure $4 \mathrm{~b}$ ). A slight increase in the degree of crystallinity was found when the fiber diameter decreased (i.e. 18 and $23 \%$ were determined for micro and nanofibers, respectively), suggesting that a higher molecular orientation was obtained from the dilute solutions chosen to produce nanofibers. X-ray diffraction patterns of PLA micro- and nanofibers loaded with triclosan (3 wt $\%)$ were different since characteristic triclosan reflections were only detected in the pattern of nanofibers (Figure 4c). Thus, drug molecules were well mixed in the PLA amorphous phase or formed independent crystalline domains depending on the fiber diameter size. Probably not all triclosan molecules could be incorporated in the nanofibers, and crystallized over their surface, in agreement with recent release studies.

Figure 5 compares the WAXD profiles of PCL, P99 and COP matrices containing PLA micro/nanofibers (i.e. after the molding process). In all cases, very intense reflections corresponding to each poly-
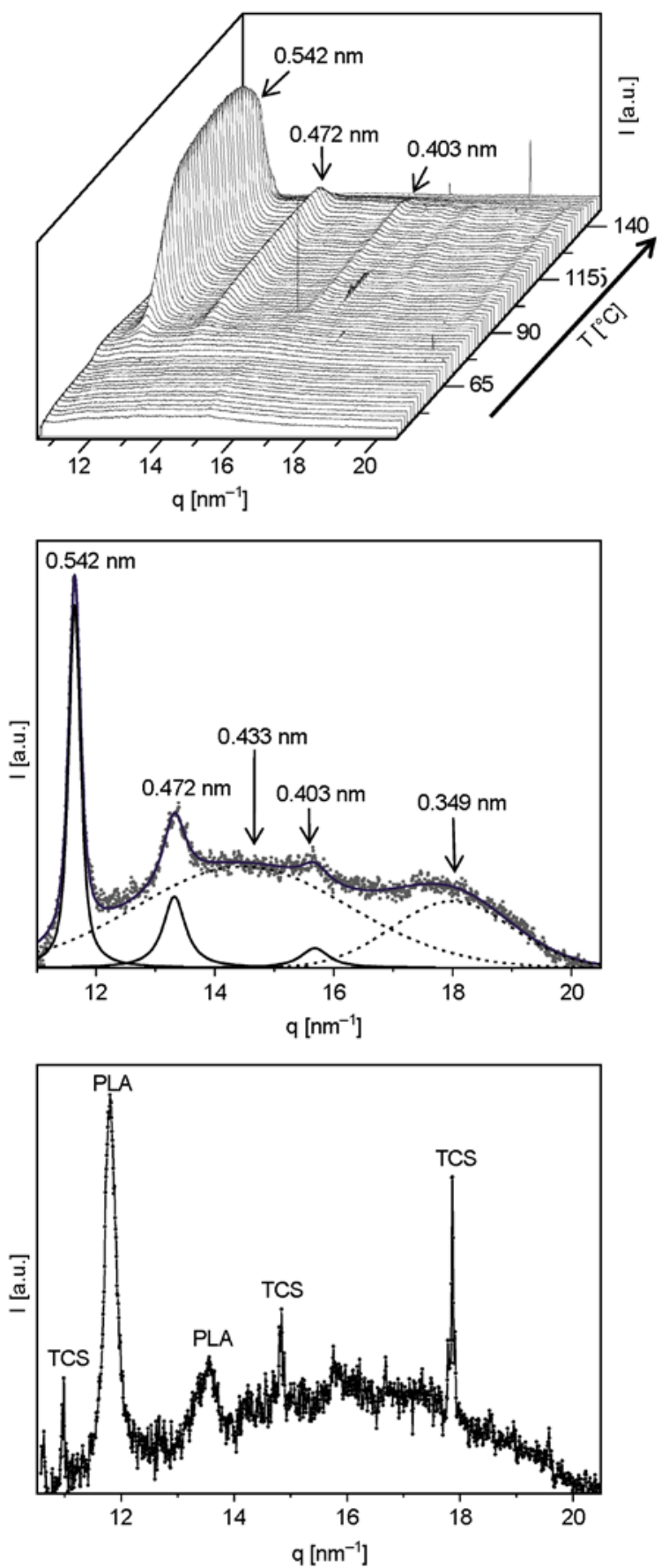

Figure 4. a) Three-dimensional WAXD profiles taken during a heating scan $\left(3^{\circ} \mathrm{C} / \mathrm{min}\right)$ of a mat constituted by electrospun PLA nanofibers. b) WAXD profile corresponding to a cold crystallized mat of PLA nanofibers. Deconvoluted amorphous halos (dashed lines) and crystalline peaks (solid lines) are indicated, along with the corresponding simulated profiles (gray solid lines). c) WAXD profile corresponding to a cold crystallized mat of PLA nanofibers loaded with triclosan where characteristic peaks of triclosan crystals (TCS) can be observed. Abscise axis corresponds to the scattering vector $\left(q=2 \pi / d_{\mathrm{B}}\right.$, where $d_{\mathrm{B}}$ is the Bragg spacing). 

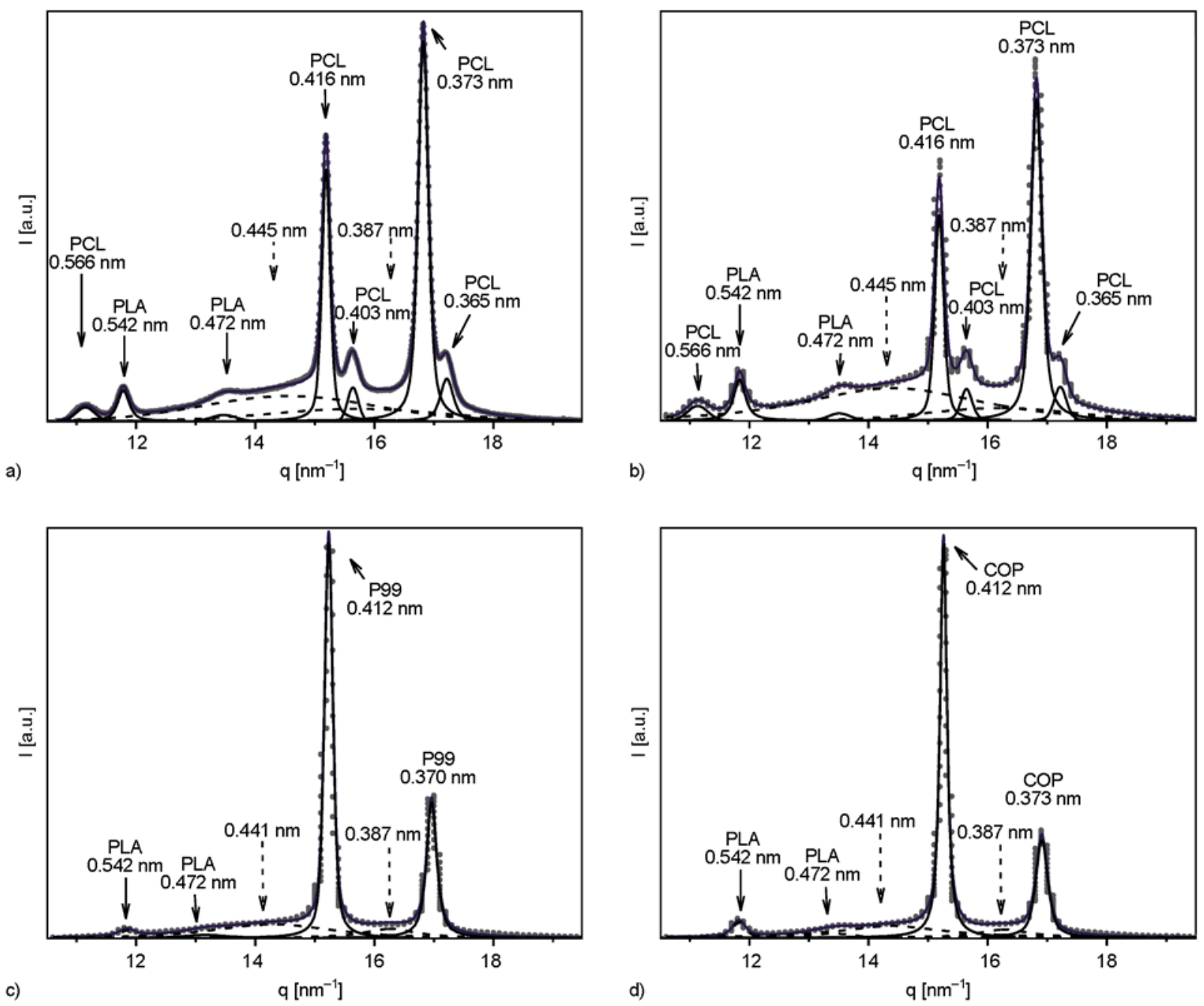

Figure 5. WAXD profiles corresponding to PCL (a, b), P99 (c) and COP (d) samples reinforced with PLA nanofibers (a, c, d) and PLA microfibers (b). Deconvoluted amorphous halos (dashed lines) and crystalline peaks (solid lines) are indicated, along with the corresponding simulated profiles (blue solid lines). Abscise axes correspond to the scattering vector ( $q=2 \pi / d_{\mathrm{B}}$, where $d_{\mathrm{B}}$ is the Bragg spacing).

ester, together with amorphous halos and the above peaks associated with cold crystallized PLA, were observed. Thus, PCL samples showed peaks at $0.566,0.416,0.403$ and $0.373 \mathrm{~nm}$, which correspond to the (102), (110), (111) and (200) reflections of polycaprolactone $[25,26]$ whereas both P99 and COP samples showed peaks at 0.412 and 0.373 $0.370 \mathrm{~nm}$, which are indicative of similar molecular packing arrangements. In all cases, the relative intensity of PLA reflections slightly increased when using nanofibers whereas the relative intensity of the polyester matrix reflections remained almost constant. The degree of crystallinity was rather similar for micro- and nanofiber assemblies since the polyester matrix was always predominant, although slight differences could be detected when only PLA reflections and amorphous halos were considered (Table 1).
The low molecular weight of P99 and COP samples gave rise to slightly higher crystallinities for assemblies based on these polyester matrices.

WAXD patterns of triclosan loaded samples did not show any peaks attributable to the drug and demonstrated that triclosan was well mixed in the PCL, P99, COP and PLA polyester matrices. Diffraction patterns of loaded and unloaded samples were rather similar although a slight decrease in the degree of crystallinity was logically detected in samples containing triclosan. It should be pointed out that PLA reflections could still be detected in all cases, indicating that the drug could not completely avoid the cold crystallization process. Table 1 summarizes the crystallinity data determined for all samples studied. 
Table 1. Estimated crystallinities from X-ray diffraction data for the different studied samples

\begin{tabular}{|c|c|c|c|c|}
\hline \multirow{2}{*}{ Sample $^{\mathrm{a}}$} & \multicolumn{4}{|c|}{ Crystallinity 100. $\mathbf{I}_{\mathrm{PLA}} /\left(\mathrm{I}_{\mathrm{PLA}}+\mathrm{I}_{\mathrm{am}}\right)^{\mathrm{b}}$} \\
\hline & $\mathbf{O} \mathbf{T}^{\mathbf{c}}$ & $3 T^{\mathrm{c}}$ & $\mathbf{O T}^{\mathrm{c}}$ & $3 T^{\mathrm{c}}$ \\
\hline PLA-N $^{d}$ & 23 & $23^{\mathrm{e}}$ & 23 & 23 \\
\hline PLA-M ${ }^{\mathrm{d}}$ & 18 & 17 & 18 & 17 \\
\hline PCL & 49 & - & - & - \\
\hline PE99 & 69 & - & - & - \\
\hline COP & 62 & - & - & - \\
\hline PCL/PLA-N/PCL & 44 & $44^{\mathrm{f}}$ & 12 & $11^{\mathrm{f}}$ \\
\hline PCL/PLA-M/PCL & 57 & $56^{\mathrm{f}}$ & 9 & $8^{\mathrm{f}}$ \\
\hline PE99/PLA-N/PE99 & 65 & $63^{\mathrm{f}}$ & 14 & $12^{\mathrm{f}}$ \\
\hline PE99/PLA-M/PE99 & 63 & $62^{\mathrm{f}}$ & 11 & $10^{\mathrm{f}}$ \\
\hline COP/PLA-N/COP & 65 & $63^{\mathrm{f}}$ & 20 & $18^{\mathrm{f}}$ \\
\hline COP/PLA-M/COP & 63 & $62^{\mathrm{f}}$ & 17 & $15^{\mathrm{f}}$ \\
\hline
\end{tabular}

${ }^{a} \mathrm{~N}$ and $\mathrm{M}$ indicate nanofibers and microfibers, respectively

${ }^{b}$ Ratio between the intensity of PLA reflections and the total intensity once the crystalline reflections of the polyester matrix were discarded. Note that amorphous halos of the matrix are still considered.

${ }^{\mathrm{c}} 0 \mathrm{~T}$ and $3 \mathrm{~T}$ denote samples containing triclosan weight percentages of 0 and $3 \%$, respectively.

${ }^{\mathrm{d}}$ Cold crystallized samples.

${ }^{e}$ Without considering diffraction peaks associated to triclosan.

${ }^{\mathrm{f}}$ Triclosan was only loaded into the PLA fibres and the percentage refers to the PLA content.

\subsection{Mechanical properties of polyester matrices reinforced with PLA micro/nanofibers}

Very few works address the development of electrospun fibers as reinforcing materials despite their high potential interest $[8,9,27]$. For example, it has been clearly demonstrated that addition of nylon 6 electrospun nanofibers to an epoxy resin enhances the stiffness and strength of the polymer matrix significantly. It has also been established that electrospun nanofibers should have advantages [28] over traditional fillers since the reinforcing effect of the former is mainly influenced by fiber size. Hence, the integrity of the polymer matrix should be enhanced by the use of fibers with nanometer dimensions instead of conventional fibers. Furthermore, the high porosity of electrospun fiber mats can result in an interpenetrating structure after their incorporation into the polymer matrix and an increase of the mechanical strength caused by the interlocking mechanism [9, 27]. Another advantage of smaller fiber diameters is that they should lead to higher tensile strengths since the fiber surface becomes dominant over the core region with bulk-like properties, and segmental motion is more constrained when dimensions approach the polymer radius of
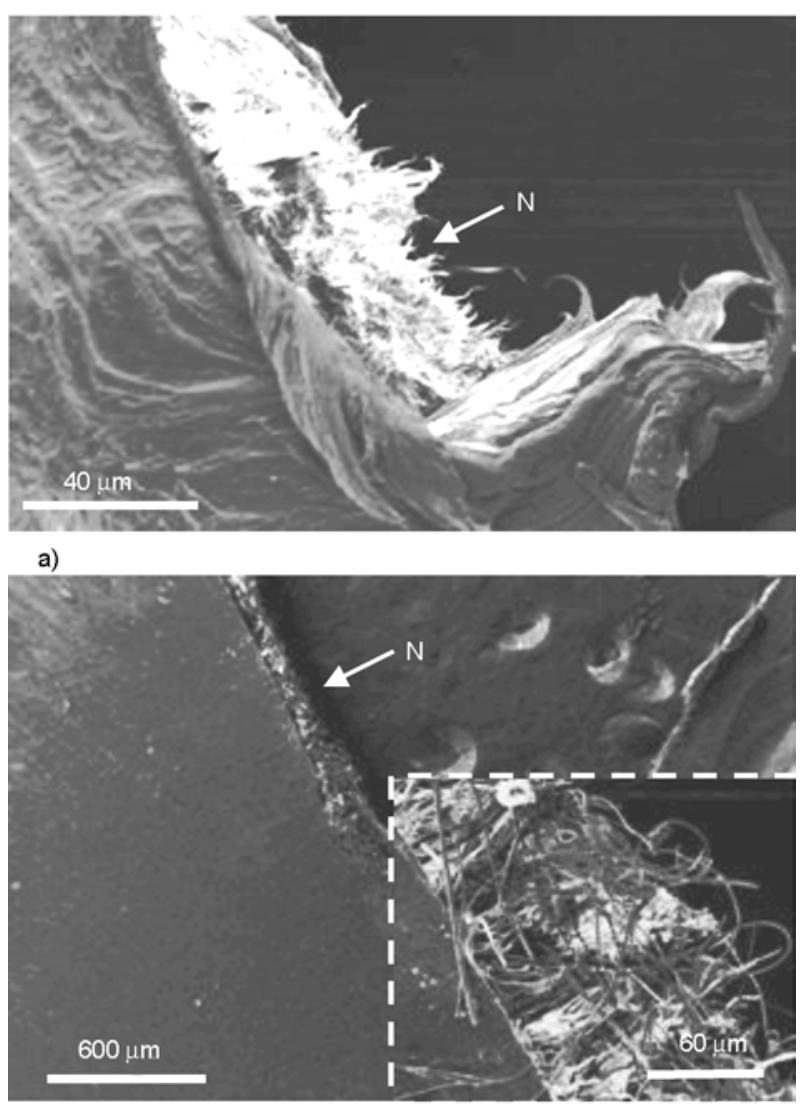

b)

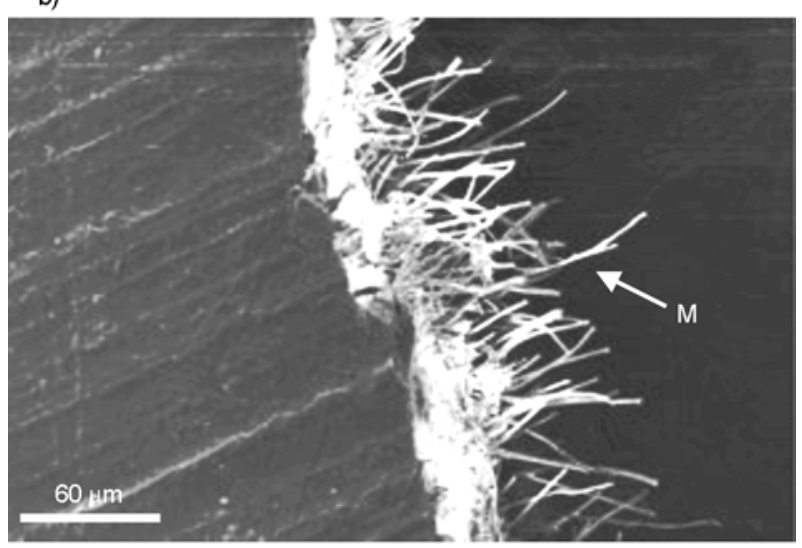

c)

Figure 6. SEM micrographs showing the fracture surface of PCL reinforced with PLA nanofibers (a), P99 reinforced with PLA nanofibers (b) and COP reinforced with PLA microfibers (c). Arrows point to PLA nanofibers $(\mathrm{N})$ and microfibers $(\mathrm{M})$.

gyration [27, 29]. Finally, it must be pointed out that oriented and stretched polymer chains in electrospun samples lead to a decrease in the tensile deformation with respect to the bulk polymer [27, 30].

Representative fracture surfaces of samples after mechanical testing show micro/nanofiber pullout (Figure 6) as well as good dispersion of fibers inside the matrix. Note also that fibers appear aligned after breaking in the direction of the applied stress, a fea- 
ture that contrasts with the structure of the inserted mats, which is characterized by a random orientation of long micro/nanofibers. Crack damage appears to start in the polyester matrix; then fibers are affected by the external force and pulled out and aligned before breaking. The strength of these fiber composites depends on the evolution of damage, which is a combination of matrix cracking, fiber fracture, fiber pullout, debonding and inelastic matrix deformation throughout the application of loading [31, 32].

Figure 7a compares the stress-strain curves obtained with the PCL matrix and PCL samples reinforced with PLA micro/nanofiber mats. Curves exhibited linear behavior at early stages of loading, which became non-linear before maximum load was reached. A drop in load followed as a result of failure initiation. Table 2 summarizes the main mechanical parameters determined for all samples studied before and after incorporation of PLA mats. Some conclusions can be drawn from these values: a) The incorporation of the reinforcing fibers clearly enhanced the stiffness of the material; consequently, the elastic modulus increased by 115 and $48 \%$ by adding the microfiber and nanofiber mats, respectively, b) The increase in the maximum tensile strength was greater when microfibers were employed (i.e. 21\%), c) The unfilled PCL matrix showed a plastic deformation that led to a high strain at break which contrasted with the reduced values determined for filled samples. Figure 7 also shows that the elastic modulus and tensile strength of triclosan loaded samples decreased slightly and significantly, respectively, probably due to a slight decrease in the

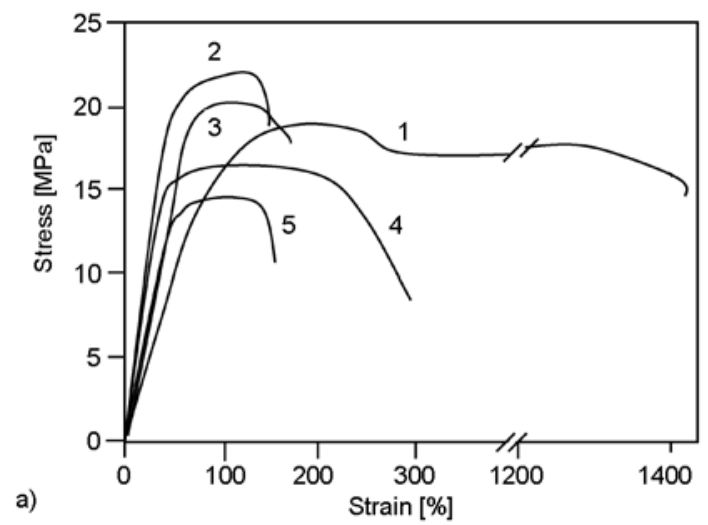

PLA crystallinity (Table 1) and a more significant reduction of the surface interactions between the matrix and added fibers.

Figure $7 \mathrm{~b}$ and Table 2 point out that properties were greatly improved by reinforcing low molecular weight polymers (i.e. P99 and COP) since these matrices had brittle fracture, low deformation at break and low tenacity. Hence, the enhancement of tensile strength became highly relevant when PLA mats were added. Note that the best results were obtained again by using fibers with micrometer diameter sizes (e.g. the modulus increased by 313 and $80 \%$ when COP was reinforced with microfiber and nanofiber mats, respectively).

In general, mechanical properties showed less improvement when nanofibers were used. This striking feature may be an inherent consequence of the fabric procedure where mats with randomly oriented fibers and different porosity were employed. Note that SEM micrographs of electrospun mats

Table 2. Mechanical properties of the different studied samples

\begin{tabular}{|l|c|c|c|}
\hline \multicolumn{1}{|c|}{ Sample $^{\mathbf{a}}$} & $\begin{array}{c}\text { Young } \\
\text { modulus } \\
{[\mathbf{M P a}]}\end{array}$ & $\begin{array}{c}\text { Maximum } \\
\text { stress } \\
{[\mathbf{M P a}]}\end{array}$ & $\begin{array}{c}\text { Strain at } \\
\text { break } \\
{[\%]}\end{array}$ \\
\hline PCL & $19 \pm 2$ & $18 \pm 2$ & $1400 \pm 80$ \\
\hline PCL/PLA-M/PCL & $41 \pm 3$ & $22 \pm 2$ & $150 \pm 10$ \\
\hline PCL/PLA-N/PCL & $28 \pm 2$ & $19 \pm 2$ & $170 \pm 10$ \\
\hline PE99 & $11 \pm 1$ & $2 \pm 0.5$ & $17 \pm 2$ \\
\hline PE99/PLA-M/PE99 & $60 \pm 4$ & $7 \pm 1$ & $10 \pm 2$ \\
\hline PE99/PLA-N/PE99 & $22 \pm 2$ & $5 \pm 1$ & $18 \pm 3$ \\
\hline COP & $15 \pm 1$ & $7 \pm 1$ & $68 \pm 6$ \\
\hline COP/PLA-M/COP & $62 \pm 3$ & $11 \pm 1$ & $17 \pm 3$ \\
\hline COP/PLA-N/COP & $27 \pm 2$ & $8 \pm 1$ & $33 \pm 3$ \\
\hline
\end{tabular}

${ }^{a} \mathrm{~N}$ and $\mathrm{M}$ indicate nanofibers and microfibers, respectively.

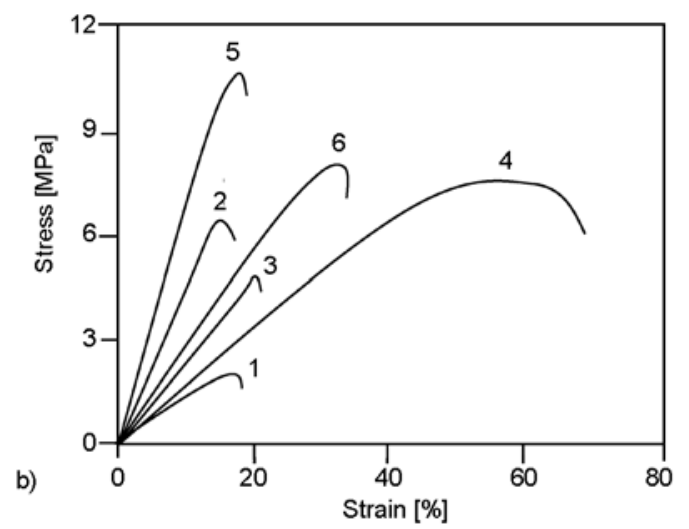

Figure 7. a) Stress-strain curves of PCL without reinforcing PLA fibers (1), with PLA microfibers $(2,4)$ and with PLA nanofibers $(3,5)$. Fibers were unloaded $(2,3)$ or loaded with $3 \mathrm{wt} \%$ of TCS $(4,5)$. b) Stress-strain curves of P99 $(1,2,3)$ and $\operatorname{COP}(4,5,6)$ samples without reinforcing PLA fibers $(1,4)$, with PLA microfibers $(2,5)$ and with PLA nanofibers $(3,6)$. 
showed a higher spacing between microfibers than between nanofibers (i.e. $5 \mu \mathrm{m}$ versus ca. $1 \mu \mathrm{m}$ as shown in Figures 2a and 2c). Thus, nanofibers gave rise to a highly compact and scarcely porous threedimensional arrangement that made it difficult to achieve optimal surface interactions with the melted polyester matrix by the subsequent molding process.

\subsection{Triclosan release from mats of PLA micro/nanofibers and polyester matrices filled with PLA micro/nanofibers}

The release rates can be calculated from experimental results by considering several theoretical models, typically first-order [33, 34], Higuchi [35, 36] and their combination [37]. The release from mat samples constituted by fibers and even from film samples usually occurred in two different steps, i.e. rapid release of molecules that should be deposited on the surface area and slow release that should involve the diffusion of molecules through the polymer matrix towards the release medium. Thus, the Higuchi (Equation (1)) and first-order (Equation (2)) models were usually combined to describe the first $(0-60 \%)$ and second $(40-100 \%)$ parts of the release, respectively:

$$
\frac{M_{\mathrm{t}}}{M_{0}}=k_{\mathrm{h}} t^{1 / 2} \quad\left(0 \leq \frac{M_{\mathrm{t}}}{M_{0}} \leq 0.6\right)
$$

$\ln \left(1-\frac{M_{\mathrm{t}}}{M_{0}}\right)=a-k_{1} t\left(0 \leq \frac{M_{\mathrm{t}}}{M_{0}} \leq 1.0\right)$

where $k_{\mathrm{h}}$ is the Higuchi release constant, $k_{1}$ is the first-order release constant, $a$ takes into account the release in the first step, $M_{\mathrm{t}}$ is the percentage of drug released at time $t$, and $M_{0}$ is the triclosan equilibrium percentage (considered as the maximum triclosan percentage that can be delivered in the medium). The assumption of the late-time Equation (2) implies that the amount of drug released at each time is proportional to the residual drug inside the dosage form.

Triclosan release in Sörensen medium [38] is characterized by the achievement of an equilibrium condition where the affinity between drug and polymer matrix plays a substantial role. This equilibrium can be modified by increasing the hydrophobicity of the release medium, which is easily achieved by adding ethanol. A volume percentage of $70 \%$ had been previously found to give similar release results to more
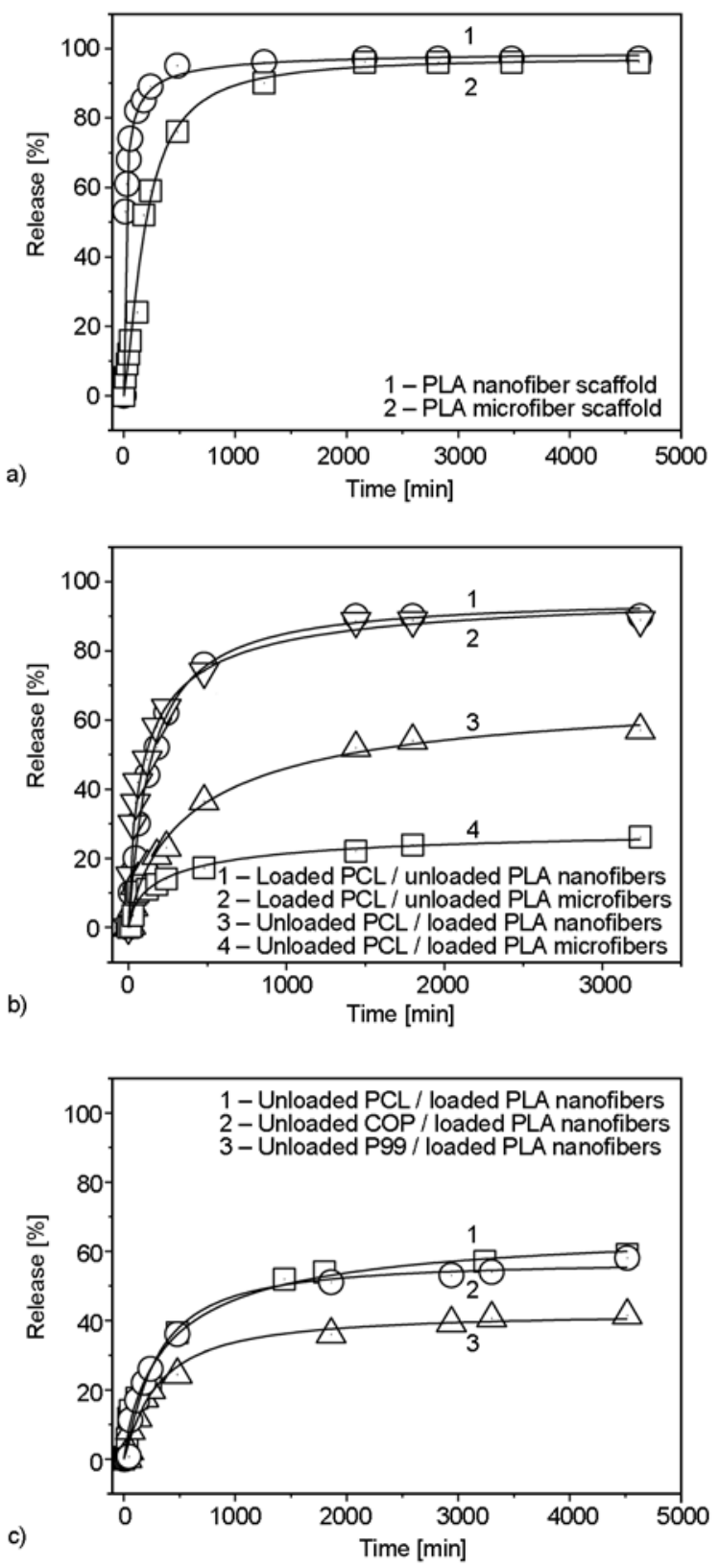

Figure 8. Comparison between cumulative triclosan release in a Sörensen/ethanol $(30 / 70 \mathrm{v} / \mathrm{v})$ medium from PLA scaffolds (a, curves: 1, PLA nanofiber; 2, PLA microfiber), differently loaded PCL samples (b, curves: 1, loaded PCL / unloaded PLA nanofibers; 2, loaded PCL / unloaded PLA microfibers; 3, unloaded PCL / loaded PLA nanofibers; 4, unloaded PCL / loaded PLA microfibers), and different polyester matrices filled with PLA microfiber mats (c, curves: 1, unloaded PCL / loaded PLA nanofibers; 2, unloaded COP / loaded PLA nanofibers; 3, unloaded P99 / loaded PLA nanofibers).

expensive serum-based media [38]. It should also be pointed out that ethanol may have some swelling effect that could facilitate the drug release. 
The cumulative triclosan release from PLA scaffolds constituted by electrospun fibers with micrometer or nanometer diameter sizes is compared in Figure $8 \mathrm{a}$. Release profiles are significantly different and indicate a logically higher rate when fibers had smaller diameter sizes since the drug should be closer to the fiber surface, decreasing the drug diffusion path length. Furthermore, a significant Burst effect was observed, in agreement with the existence of triclosan crystalline domains probably located on the fiber surface, as revealed by WAXD data. Table 3 compares the release constants for the two samples when the combined model was used. It is clear that both $k_{\mathrm{h}}$ and $k_{1}$ release constants decreased drastically for the electrospun microfibers. It should be noted that, in this case, $k_{1}$ became close to the value previously determined for similar PLA scaffolds (i.e. 0.09 and $0.08 \mathrm{~min}^{-1}$ ) [24]. Release data also indicate that all the triclosan was practically released in the selected medium and only an increase in the time required for complete delivery was observed when the diameter was increased (i.e. 700 and $1600 \mathrm{~min}$ for the nanofibers and microfibers with average diameters of 2.56 and $0.350 \mu \mathrm{m}$ ). An asymptotic release percentage was clearly detected when PCL fabric samples were employed (Figure 8b), suggesting that equilibrium conditions were achieved when the medium was not renewed or that some molecules were entrapped in the dosage form. No remaining percentage was observed for PLA scaffolds but it should be taken into account that release behaviors could not be strictly compared due to the different texture (i.e. compact or porous) and geometry of samples. However, it should be reminded that previous results on similar PCL scaffold samples [24] showed greater affinity of triclosan with the more hydrophobic PCL polymer and a lower final release percentage when PLA scaffolds were progressively enriched with PCL. As can be seen in Table 3 and Figure 8b, very few differences were found when triclosan loaded PCL matrices were reinforced with PLA micro/nanofibers. Nevertheless, the kinetic constant values (Table 3) indicate that the initial and faster release step was slightly disfavored by addition of nanofibers, suggesting greater difficulty for triclosan molecules to diffuse through the nanofilled polymer matrix.

Release behavior changed drastically when the drug was only loaded into the reinforcing PLA micro/ nanofibers, as shown in Figure 8b. First, a small time period $\left(t_{0}\right.$ values summarized in Table 3$)$ seemed to be required for the start of the release. Consequently, the experimental data can only be fitted to a Higuchi behavior after the observed 'induction' time. Apparently, drug molecules need a significant time period to reach the surface of the polymer matrix from the filled fibers. Second, the release initially progressed at a moderate rate (note that $k_{\mathrm{h}}$ was always significantly lower than the values calculated for samples with the drug directly loaded into the polymer matrix). Third, the release rate also decreased during the second step defined by a firstorder kinetics. It is important to point out that the release tended to an asymptotic value which was clearly lower than that found for samples with the

Table 3. Correlation coefficients and release rate constant for the fitting to the combined model of release profiles corresponding to the indicated samples loaded with $3 \mathrm{wt} \%$ of triclosan and delivered in a Sörensen/ethanol medium $(30 / 70 \mathrm{v} / \mathrm{v})$

\begin{tabular}{|c|c|c|c|c|c|c|}
\hline \multirow{2}{*}{ Sample } & \multicolumn{2}{|c|}{ Early-Time } & \multicolumn{2}{|c|}{ Late-Time } & \multirow{2}{*}{$\begin{array}{c}\mathbf{t}_{0}{ }^{\mathrm{a}} \\
{[\mathrm{min}]}\end{array}$} & \multirow{2}{*}{$\begin{array}{c}\mathbf{t}_{\mathbf{f}}^{\mathbf{b}^{2}} \\
{[\mathrm{~min}]}\end{array}$} \\
\hline & $\mathbf{k}_{\mathrm{h}}\left[\mathrm{h}^{-0.5}\right]$ & $\mathbf{r}$ & $\mathbf{k}_{1}\left[\mathbf{h}^{-1}\right]$ & $\mathbf{r}$ & & \\
\hline PLA Nano $^{c}$ & 0.957 & 0.957 & 0.378 & 0.991 & 0 & 700 \\
\hline PLA Micro $^{c}$ & 0.160 & 0.977 & 0.090 & 0.994 & 0 & 1600 \\
\hline PCL + PLA Nano ${ }^{\mathrm{d}}$ & 0.320 & 0.981 & 0.198 & 0.996 & 0 & 2500 \\
\hline PCL + PLA Micro ${ }^{d}$ & 0.379 & 0.976 & 0.084 & 0.986 & 0 & 2500 \\
\hline PCL + PLA Nano ${ }^{\mathrm{e}}$ & 0.159 & 0.998 & 0.072 & 0.996 & 15 & 5000 \\
\hline PCL + PLA Micro ${ }^{e}$ & 0.139 & 0.980 & 0.060 & 0.993 & 30 & 6000 \\
\hline $\mathrm{COP}+\mathrm{PLA} \mathrm{Nano}^{\mathrm{e}}$ & 0.265 & 0.999 & 0.036 & 0.990 & 45 & 6300 \\
\hline P99 + PLA Nano ${ }^{\mathrm{e}}$ & 0.215 & 0.970 & 0.036 & 0.995 & 45 & 6300 \\
\hline
\end{tabular}

${ }^{a}$ Time necessary to initiate the Higuchi release.

${ }^{\mathrm{b}}$ Time necessary to reach a constant release.

'Scaffold samples.

${ }^{\mathrm{d}}$ Triclosan was only loaded in the PCL matrix.

${ }^{\mathrm{e}}$ Triclosan was only loaded in the PLA electrospun fibers. 
drug directly loaded into the matrix and that the final release percentage was lower when the drug was loaded into PLA microfibers instead of PLA nanofibers. This large difference between the three loaded samples cannot be justified by the achievement of equilibrium conditions since practically the same value should be deduced. Results suggest that triclosan was entrapped in the PLA fibers and could not effectively diffuse through the PCL matrix at an appreciable rate. The amount of retained triclosan logically increased with the fiber diameter. As a result, a lower cumulative release percentage was found for samples containing microfibers. The initial fast release step may be a consequence of the preparation method since at the molding temperature some diffusion from PLA nano/microfibers to the melted PCL matrix could have occurred, and then (i.e. at room temperature) diffusion was clearly hindered.

Table 4. Contact angle $(\Theta)$ for the polyester films (PCL, $\mathrm{P} 99, \mathrm{COP})$ and polyester matrices reinforced with mats of PLA microfibers (M) amd nanofibers $(\mathrm{N})$.

\begin{tabular}{|l|l|}
\hline \multicolumn{1}{|c|}{ Sample } & $\boldsymbol{\Theta}\left[^{\circ}\right]^{\mathrm{a}}$ \\
\hline PCL & $81.4 \pm 2.5$ \\
\hline PCL/PLA-M/PCL & $77.3 \pm 5.3$ \\
\hline PCL/PLA-N/PCL & $66.2 \pm 4.9^{\mathrm{b}}$ \\
\hline P99 & $90.5 \pm 6.2^{\mathrm{c}}$ \\
\hline P99/PLA-M/P99 & $80.5 \pm 1.9^{\mathrm{b}}$ \\
\hline P99/PLA-N/P99 & $79.4 \pm 3.4^{\mathrm{b}}$ \\
\hline COP & $87.7 \pm 6.0^{\mathrm{c}}$ \\
\hline COP/PLA-M/COP & $78.8 \pm 3.9^{\mathrm{b}}$ \\
\hline COP/PLA-N/COP & $74.4 \pm 0.8^{\mathrm{b}}$ \\
\hline
\end{tabular}

${ }^{a}$ Average values and standard deviation over six measurements.

${ }^{\text {b}}$ Tukey test, $p<0.05$, composite vs. polymer film.

${ }^{\mathrm{c}}$ Tukey test, $p<0.05$, reference film (PCL) vs. polymer film.

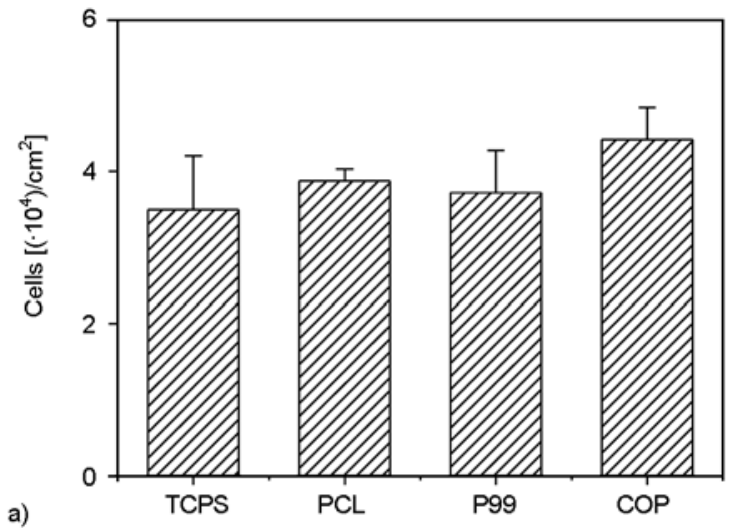

Figure 8c shows that release behavior depended on the hydrophobicity of the polymer matrix, which could be evaluated by the contact angle measurements summarized in Table 4. Hence, the release rate constants (Table 3 ) and even the asymptotic release value decreased with hydrophobicity, which depended on the methylene content of the repeat unit and increased in the order P99 $>$ COP $>$ PCL.

Samples containing triclosan loaded micro/nanofibers appear highly interesting since a relatively fast release is observed at the beginning of the delivery process; hence, a rapid antimicrobial effect should be expected; as found from the release of the similar systems [24, 38]. Furthermore, a considerable amount of drug could be retained, and therefore pharmacological activity may be observed again when degradation of the polymer matrix reaches a considerable level. Furthermore, the amount of retained drug can be tuned by varying the fiber diameter size and the hydrophobic character of the polymer matrix.

\subsection{Biocompatibility of polyester matrices reinforced with PLA micro/nanofibers}

The adhesion assay was carried out on the film surfaces of the processed materials to evaluate the quality of the new polyesters (P99 and COP) as substrates for cell attachment. These polymers showed slightly improved adhesion in comparison to TCPS or PCL (used as a reference). The increase was more evident for COP, although the number of cells adhered to the studied surfaces was not statistically different (Figure 9a). Figure 10 qualitatively compares the aspect of the cells adhered to the selected

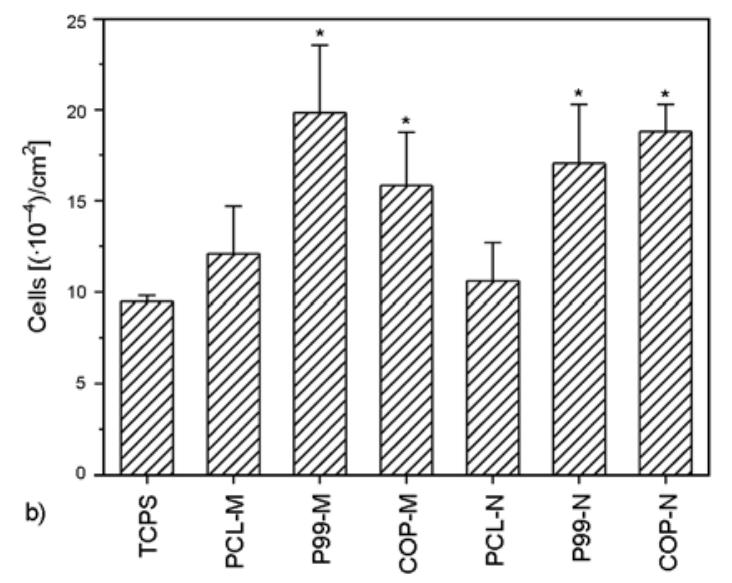

Figure 9. a) Attachment of HEp-2 cells to the surface of PCL, P99 and COP samples. b) Cell proliferation on TCPS and the above polyesters loaded with PLA microfibers (M) or PLA nanofibers (N) after 7 days of culture. The vertical bars are the average values $\pm \mathrm{SD}$. $(*) p<0.05$ vs control sample (TCPS or surface plate), $t$-Student test. 
polyester surfaces. Cells spread extensively on the PCL and COP surfaces (Figures 10a-b and 10e-f, respectively) whereas very little spreading was detected on the P99 surface where cells clearly had a more rounded morphology (Figure 10c-d). This morphology-based approach suggests again that the COP surface supports cell adhesion better.

Cell proliferation assays were conducted to evaluate the three reinforced polyester matrices, which were characterized by a three-dimensional array

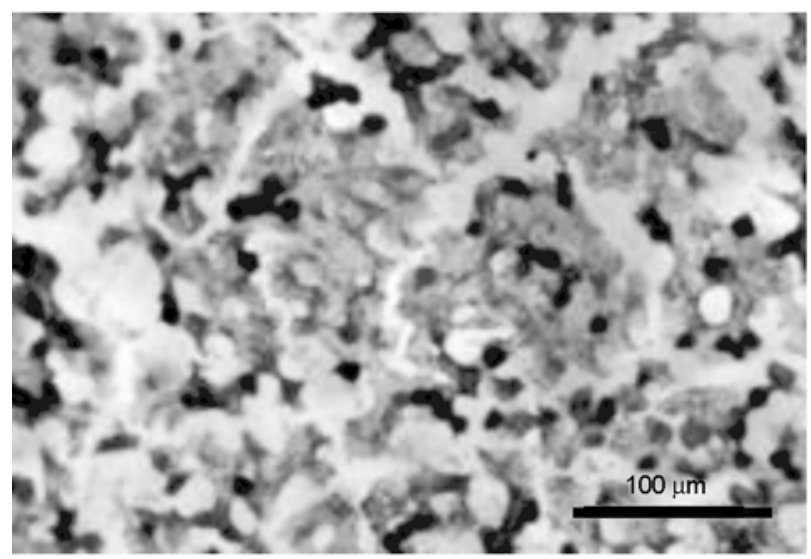

a)

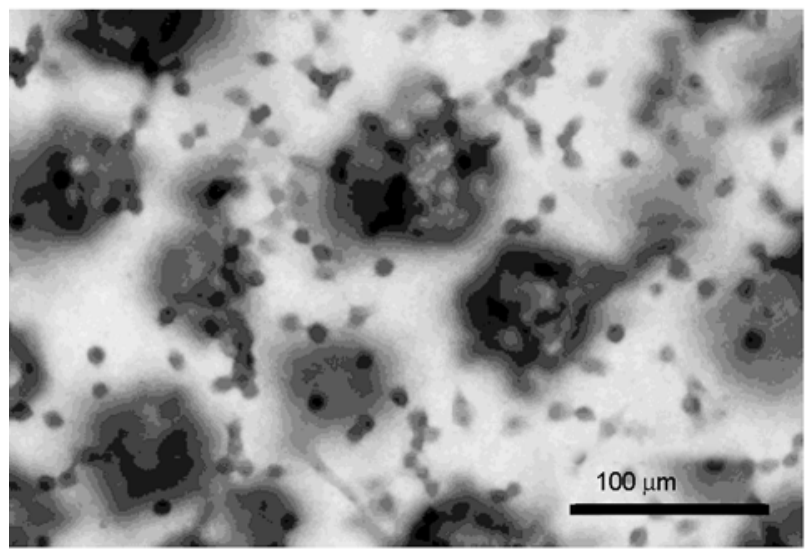

c)

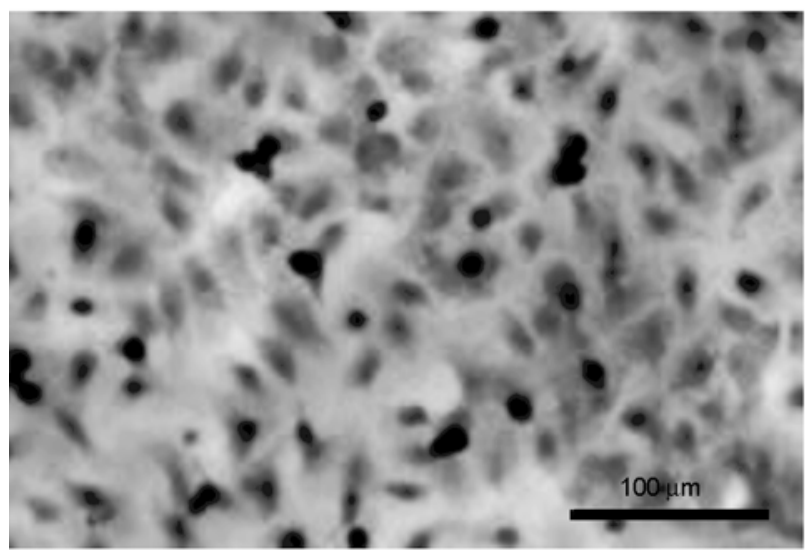

e) that may be a first approximation to more conventional scaffolds. Figure $9 \mathrm{~b}$ shows the results of the HEp-2 cell culture for 7 days on all materials studied. The number of cells in the PCL matrix reinforced with mats constituted by PLA micro/nanofibers (PCL-M and PCL-N, respectively) suggested a similar growth to that observed for the control (TCPS). However, cell growth in reinforced P99 and COP matrices was significantly higher than in the control $(p<0.05)$ and in PCL samples, although

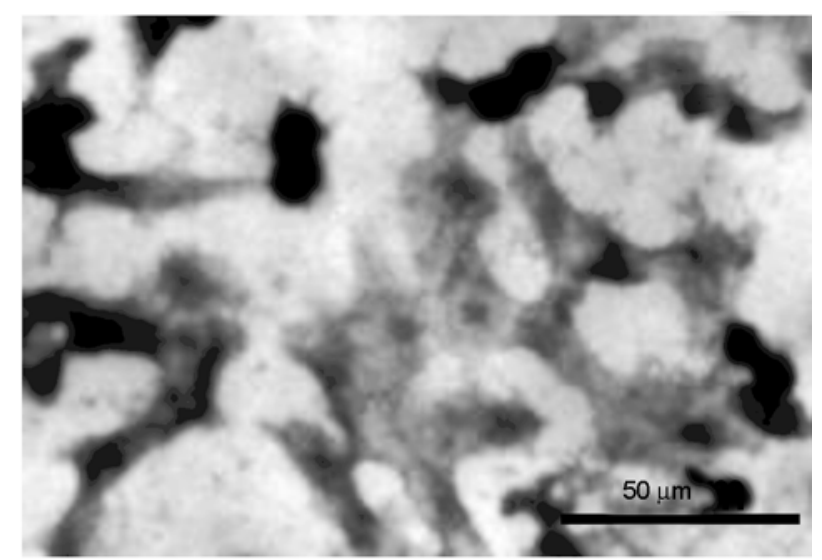

b)

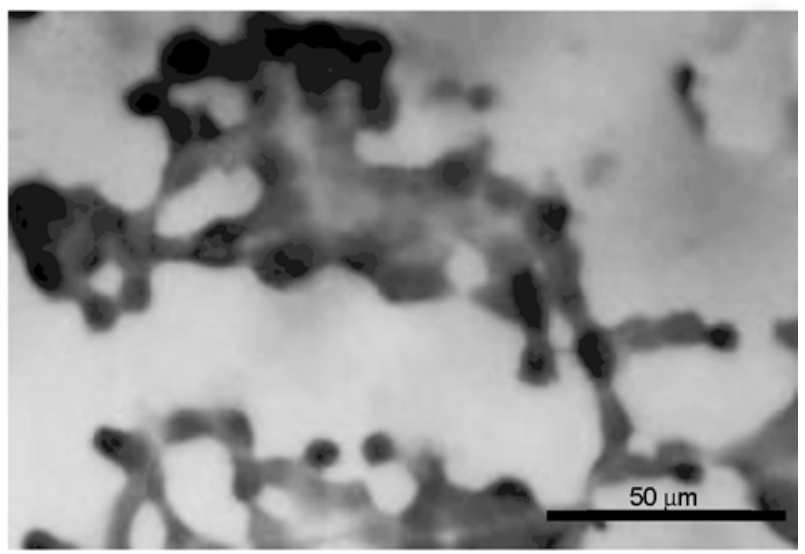

d)

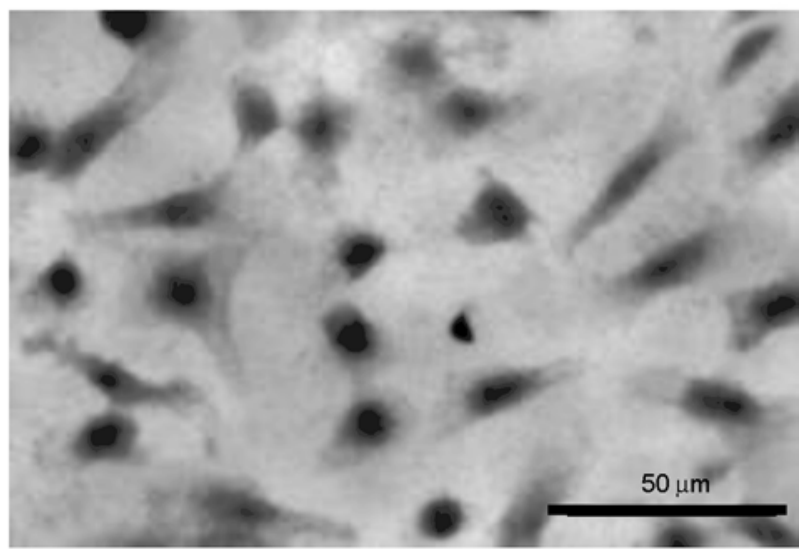

f)

Figure 10. Optical micrographs showing the morphology of HEp-2 cells adhered to PCL (a, b), P99 (c, d) and COP (e, f) substrates 
in this case the difference did not reach a significant statistical value. These results can be justified considering that all polyester samples corresponded to three-dimensional architectures while the TCPS control corresponded to a cell growth in a twodimensional system, and consequently with a smaller available surface. It should also be highlighted that no significant differences were detected by using reinforcing mats constituted by PLA micro/nanofibers. Note that all surfaces studied had a similar number of cells in the initial event of adhesion. Differences in their colonization can therefore be directly related to the structure and properties of materials. The present study indicates that all polyester fabrics evaluated appear highly biocompatible because they supported cell growth well.

\section{Conclusions}

Mats constituted by PLA micro/nanofibers can be easily prepared by changing only the polymer concentration of the solution used in the electrospinning process. These fibers showed a high molecular orientation that was more significant with smaller fiber diameter sizes and favored the cold crystallization process.

Fabrics constituted by PLA mats inserted in polyesters with lower melting points had high crystallinity when prepared by a molding and slow cooling process that allowed both PLA cold crystallization and hot crystallization of the polyester matrix. These fabrics showed also a significant enhancement of both elastic modulus and tensile strength respect to the corresponding polyester matrices. Materials were biocompatible and were successful to provide a sustained drug release when the pharmacological agent was directly loaded into the reinforcing PLA mat. Applications concerning an enlargement of antimicrobial effect (e.g. by incorporation of triclosan) can be considered.

\section{Acknowledgements}

This research has been supported by grants from MCYT/ FEDER and AGAUR (MAT2009-11503, 2009SGR-1208). We are grateful to Drs. François Fauth and Ana Labrador of the CRG BM16 beamline staff of CELLS (Consortium for the Exploitation of the Synchrotron Light Laboratory). We are also grateful to Dr. Trifon Trifonov for FIB micrographs.

\section{References}

[1] Boudriot U., Dersch R., Greiner A., Wendorff J. H.: Electrospinning approaches toward scaffold engineering - A brief overview. Artificial Organs, 30, 785-792 (2006).

DOI: $10.1111 / \mathrm{j} .1525-1594.2006 .00301 . \mathrm{x}$

[2] Katti D. S., Robinson K. W., Ko F. K., Laurencin C. T.: Bioresorbable nanofiber-based systems for wound healing and drug delivery: Optimization of fabrication parameters. Journal of Biomedical Materials Research Part B: Applied Biomaterials, 70, 286-296 (2004). DOI: $10.1002 / \mathrm{jbm} . b .30041$

[3] Li S., Patel S., Hashi C., Huang N. F., Kurpinski K.: Biomimetic scaffolds. U.S. Patent 20070269481, USA (2007).

[4] Reneker D. H., Yarin A. L., Fong H., Koombhongse S.: Bending instability of electrically charged liquid jets of polymer solutions in electrospinning. Journal of Applied Physics, 87, 4531-4547 (2000). DOI: $10.1063 / 1.373532$

[5] Yarin A. L., Koombhongse S., Reneker D. H.: Bending instability in electrospinning of nanofibers. Journal of Applied Physics, 89, 3018-3027 (2001). DOI: $10.1063 / 1.1333035$

[6] Dror Y., Salalha W., Khalfin R. L., Cohen Y., Yarin A. L., Zussman E.: Carbon nanotubes embedded in oriented polymer nanofibers by electrospinning. Langmuir, 19, 7012-7020 (2003).

DOI: $10.1021 / 1 \mathrm{a} 034234 \mathrm{i}$

[7] Stephens J. S., Chase D. B., Rabolt J. F.: Effect of the electrospinning process on polymer crystallization chain conformation in nylon-6 and nylon-12. Macromolecules, 37, 877-881 (2004).

DOI: $10.1021 / \mathrm{ma} 0351569$

[8] Bergshoef M. M., Vancso G. J.: Transparent nanocomposites with ultrathin, electrospun nylon-4,6 fiber reinforcement. Advanced Materials, 11, 1362-1365 (1999). DOI: 10.1002/(SICI)1521-4095(199911)11:16<1362:: AID-ADMA1362>3.0.CO;2-X

[9] Fong H.: Electrospun nylon 6 nanofiber reinforced BIS-GMA/TEGDMA dental restorative composite resins. Polymer, 45, 2427-2432 (2004).

DOI: $10.1016 /$ j.polymer.2004.01.067

[10] Pinho E. D., Martins A., Araújo J. V., Reis R. L., Neves N. M.: Degradable particulate composite reinforced with nanofibres for biomedical applications. Acta Biomaterialia, 5, 1104-1114 (2009). DOI: $10.1016 /$ j.actbio.2008.11.018

[11] Grafahrend D., Heffels K-H., Beer M. V., Gasteier P., Möller M., Boehm G., Dalton P. D., Groll J.: Degradable polyester scaffolds with controlled surface chemistry combining minimal protein adsorption with specific bioactivation. Nature Materials, 10, 67-73 (2011). DOI: $\underline{\text { 10.1038/NMAT2904 }}$ 
[12] Jones R. D., Jampani H. B., Newman J. L., Lee A. S.: Triclosan: A review of effectiveness and safety in health care settings. American Journal of Infection Control, 28, 184-196 (2000).

DOI: 10.1016/S0196-6553(00)90027-0

[13] Södergård A., Stolt M.: Properties of lactic acid based polymers and their correlation with composition. Progress in Polymer Science, 27, 1123-1163 (2002). DOI: 10.1016/S0079-6700(02)00012-6

[14] Middleton J. C., Tipton A. J.: Synthetic biodegradable polymers as orthopedic devices. Biomaterials, 21, 23352346 (2000).

DOI: $10.1016 / \mathrm{S} 0142-9612(00) 00101-0$

[15] Pouget E., Almontassir A., Casas M. T., Puiggalí J.: On the crystalline structures of poly(tetramethylene adipate). Macromolecules, 36, 698-705 (2003).

DOI: $10.1021 / \mathrm{ma} 0214052$

[16] Almontassir A., Gestí S., Franco L., Puiggalí J.: Molecular packing of polyesters derived from 1,4butanediol and even aliphatic dicarboxylic acids. Macromolecules, 37, 5300-5309 (2004).

DOI: $10.1021 / \mathrm{ma} 0499391$

[17] Gestí S., Casas M. T., Puiggalí J.: Single crystal morphology and structural data of a series of polyesters derived from 1,8-octanediol. European Polymer Journal, 44, 2295-2307 (2008).

DOI: $10.1016 /$ j.eurpolymj.2008.04.049

[18] Lim C. T., Tan E. P. S., Ng S. Y.: Effects of crystalline morphology on the tensile properties of electrospun polymer nanofibers. Applied Physics Letters, 92, 141908/1-141908/3 (2008).

DOI: $10.1063 / 1.2857478$

[19] Hurrell S., Cameron R. E.: The effect of initial polymer morphology on the degradation and drug release from polyglycolide. Biomaterials, 23, 2401-2409 (2002).

DOI: $10.1016 / \mathrm{S} 0142-9612(01) 00376-3$

[20] Miyajima M., Koshika A., Okada J., Ikeda M., Nishimura K.: Effect of polymer crystallinity on papaverine release from poly(L-lactic acid) matrix. Journal of Controlled Release, 49, 207-215 (1997). DOI: 10.1016/S0168-3659(97)00081-3

[21] de Santis P., Kovacs A. J.: Molecular conformation of poly(S-lactic acid). Biopolymers, 6, 299-306 (1968). DOI: 10.1002/bip.1968.360060305

[22] Hoogsteen W., Postema A. R., Pennings A. J., Ten Brinke G., Zugenmaier P.: Crystal structure, conformation and morphology of solution-spun poly(L-lactide) fibers. Macromolecules, 23, 634-642 (1990).

DOI: $\underline{10.1021 / \mathrm{ma} 00204 \mathrm{a} 041}$

[23] Zeng J., Chen X., Liang Q., Xu X., Jing X.: Enzymatic degradation of poly(L-lactide) and poly( $\varepsilon$-caprolactone) electrospun fibers. Macromolecular Bioscience, 4, 1118-1125 (2004).

DOI: $10.1002 /$ mabi.200400092
[24] del Valle L. J., Camps R., Díaz A., Franco L., Rodríguez-Galán A., Puiggalí J.: Electrospinning of polylactide and polycaprolactone mixtures for preparation of materials with tunable drug release properties. Journal of Polymer Research, 18, 1903-1917 (2011).

DOI: 10.1007/s10965-011-9597-3

[25] Chatani Y., Okita Y., Tadokoro H., Yamashita Y.: Structural studies of polyesters. 3. Crystal structure of poly-epsilon-caprolactone. Polymer Journal, 1, 555562 (1970).

[26] Iwata T., Doi Y.: Morphology and enzymatic degradation of poly( $\varepsilon$-caprolactone) single crystals: Does a polymer single crystal consist of micro-crystals? Polymer International, 51, 852-858 (2002).

DOI: $10.1002 /$ pi.858

[27] Baji A., Mai Y-W., Wong S-C., Abtahi M., Chen P.: Electrospinning of polymer nanofibers: Effects on oriented morphology, structures and tensile properties. Composites Science and Technology, 70, 703-718 (2010).

DOI: 10.1016/j.compscitech.2010.01.010

[28] Tian M., Gao Y., Liu Y., Liao Y., Xu R., Hedin N. E., Fong H.: Bis-GMA/TEGDMA dental composites reinforced with electrospun nylon 6 nanocomposite nanofibers containing highly aligned fibrillar silicate single crystals. Polymer, 48, 2720-2728 (2007).

DOI: $10.1016 /$ j.polymer.2007.03.032

[29] Arinstein A., Burman M., Gendelman O., Zussman E.: Effect of supramolecular structure on polymer nanofibre elasticity. Nature Nanotechnology, 2, 59-62 (2007). DOI: $10.1038 /$ nnano.2006.172

[30] Lu J-W., Zhang Z-P., Ren X-Z., Chen Y-Z., Yu J., Guo $Z-X$.: High-elongation fiber mats by electrospinning of polyoxymethylene. Macromolecules, 41, 37623764 (2008).

DOI: $10.1021 / \mathrm{ma} 702881 \mathrm{k}$

[31] Kroll L., Hufenbach W.: A physically based failure criterion for laminated composites. Mechanics of Composite Materials, 35, 277-284 (1999). DOI: $10.1007 / \mathrm{BF} 02259715$

[32] Brauer D. S., Rüssel C., Vogt S., Weisser J., Schnabelrauch M.: Degradable phosphate glass fiber reinforced polymer matrices: Mechanical properties and cell response. Journal of Materials Science: Materials in Medicine, 19, 121-127 (2008).

DOI: $10.1007 / \mathrm{s} 10856-007-3147-\mathrm{x}$

[33] Gibaldi M., Feldman S.: Establishment of sink conditions in dissolution rate determinations. Theoretical considerations and application to nondisintegrating dosage forms. Journal of Pharmaceutical Sciences, 56, 1238-1242 (1967).

DOI: $10.1002 / j p s .2600561005$

[34] Wagner J. G.: Interpretation of percent dissolved-time plots derived from in vitro testing of conventional tablets and capsules. Journal of Pharmaceutical Sciences, 58, 1253-1257 (1969). DOI: $10.1002 / j p s .2600581021$ 
[35] Higuchi T.: Rate of release of medicaments from ointment bases containing drugs in suspension. Journal of Pharmaceutical Sciences, 50, 874-879 (1961).

DOI: $10.1002 / j p s .2600501018$

[36] Higuchi T.: Mechanism of sustained-action medication. Theoretical analysis of rate of release of solid drugs dispersed in solid matrices. Journal of Pharmaceutical Sciences, 52, 1145-1149 (1963).

DOI: $10.1002 / j p s .2600521210$
[37] Baker R. W.: Controlled release of biologically active agents. Wiley, New York (1987).

[38] Zurita R., Puiggalí J., Rodríguez-Galán A.: Triclosan release from coated polyglycolide threads. Macromolecular Bioscience, 6, 58-69 (2006).

DOI: $\underline{10.1002 / \mathrm{mabi} .200500147}$ 